\title{
Antineoplastic Immunomodulating Agent
}

National Cancer Institute

\section{Source}

National Cancer Institute. Antineoplastic Immunomodulating Agent. NCI Thesaurus.

Code C129820.

Any agent that is capable of modulating the immune system in order to exert

antineoplastic effects. Antineoplastic immunomodulating agents either activate the

immune system, restore certain immune system activators or abrogate

immunosuppression. 